\title{
A Finite Model-Theoretical Proof of a Property of Bounded Query Classes within $P H$
}

\author{
Leszek Aleksander Kołodziejczyk \\ Institute of Philosophy \\ Warsaw University
}

Krakowskie Przedmieście 3, 00-047 Warsaw, Poland

e-mail: 1.kolodziejczyk@zodiac.mimuw.edu.pl

April 29th, 2004

\begin{abstract}
We use finite model theory (in particular, the method of FM-truth definitions, introduced in [MM01] and developed in [K04], and a normal form result akin to those of [Ste93] and [G97]) to prove:

Let $m \geq 2$. Then:

(A) If there exists $k$ such that $N P \subseteq \Sigma_{m} T I M E\left(n^{k}\right) \cap \Pi_{m} T I M E\left(n^{k}\right)$, then for every $r$ there exists $k_{r}$ such that $P^{N P\left[n^{r}\right]} \subseteq \Sigma_{m} T I M E\left(n^{k_{r}}\right) \cap$ $\Pi_{m} T I M E\left(n^{k_{r}}\right)$

(B) If there exists a superpolynomial time-constructible function $f$ such that $N T I M E(f) \subseteq \Sigma_{m}^{p} \cap \Pi_{m}^{p}$, then additionally $P^{N P\left[n^{r}\right]} \subsetneq$ $\Sigma_{m}^{p} \cap \Pi_{m}^{p}$.

This strengthens a result by Mocas ([M96]) that for any $r, P^{N P\left[n^{r}\right]} \subsetneq$ $N E X P$.

In addition, we use FM-truth definitions to give a simple sufficient condition for the $\Sigma_{1}^{1}$ arity hierarchy to be strict over finite models.

It is widely believed that the polynomial hierarchy is properly contained in the nondeterministic exponential time class $N E X P$. However, even a fairly
\end{abstract}


restricted fragment of the polynomial hierarchy $-P^{N P}$, the class of problems solvable in polynomial time by a deterministic machine with access to an $N P$ oracle - has not been proven to be smaller than NEXP. Moreover, the problem whether $P^{N P}$ equals $N E X P$ seems difficult, as it is known to have contradictory relativizations (see [BT94] or [BFFT01] for an oracle under which $\left.P^{N P}=N E X P\right)$.

The largest parts of $P H$ currently known to be separated from $N E X P$ are the bounded query fragments of $P^{N P}$. In [FLZ92], it was shown that

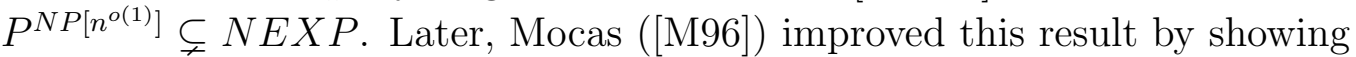
that for any fixed $r, P^{N P\left[n^{r}\right]}$ is also a proper subclass of $N E X P$.

In the present paper, we prove the following theorem:

Theorem 0.1. Let $m \geq 2$. Then:

(A) If there exists $k$ such that $N P \subseteq \Sigma_{m} T I M E\left(n^{k}\right) \cap \prod_{m} T I M E\left(n^{k}\right)$, then for every $r$ there exists $k_{r}$ such that $P^{N P\left[n^{r}\right]} \subseteq \Sigma_{m} T I M E\left(n^{k_{r}}\right) \cap \prod_{m} T I M E\left(n^{k_{r}}\right)$;

(B) If there exists a superpolynomial time-constructible function $f$ such that $N T I M E(f) \subseteq \Sigma_{m}^{p} \cap \prod_{m}^{p}$, then additionally $P^{N P\left[n^{r}\right]} \subsetneq \Sigma_{m}^{p} \cap \Pi_{m}^{p}$.

Note that the theorem can be interpreted as a strengthening of Mocas' result, which immediately follows from either $(\mathrm{A})$ or $(\mathrm{B})$. Indeed, consider e.g. (B) and assume that $P^{N P\left[n^{r}\right]}=N E X P$. Then the hypothesis of (B) is satisfied for $m=2$, so $P^{N P\left[n^{r}\right]} \subsetneq \Sigma_{2}^{p} \cap \Pi_{2}^{p} \subseteq N E X P=P^{N P\left[n^{r}\right]}$. Contradiction.

The proof uses finite model theory. We show that any $P^{N P\left[n^{r}\right]}$ property of finite models can be expressed by a second order sentence in a particular normal form, very similar to normal forms studied in [Ste93] and [G97]. We then show that if the assumption of (A) holds for a given $m$, then there is a $\Sigma_{m}^{1}$ FM-truth definition (in the sense of M. Mostowski; see [MM01]) for the class of sentences in the normal form, from which the conclusion of (A) follows via a result of $[\mathrm{K} 04]$ and the fact that $P^{N P\left[n^{r}\right]}$ is closed under complementation. (B) is established in a similar way.

The paper is divided into four sections. After the preliminary section 1, we prove (A) of theorem 0.1 in section 2 and (B) in section 3. Section 4 offers some generalizations, remarks and related results, in particular a simple sufficient condition for the arity hierarchy of $\Sigma_{1}^{1}$ to be strict in finite models. 


\section{Preliminaries}

All models are finite with built-in arithmetic. In other words, the universe of a model $\mathbf{M}$ is always an initial segment $M=\{0, \ldots, M-1\}$ of the natural numbers, and the vocabulary $\sigma$ always contains a fixed arithmetical subvocabulary $\sigma_{0}$ (consisting, say, of the predicates,$+ \times, \leq$ and the constants $0, M A X)$ whose symbols are interpreted in the standard way (e.g. $+(x, y, z)$ holds in a model iff $x+y=z$ in $\mathbb{N}$ ).

Vocabularies are finite and relational, although individual constants are allowed. A model of vocabulary $\sigma$ is referred to as a $\sigma$-model.

A logic is any function $\mathcal{L}$ which assigns to a vocabulary $\sigma$ a decidable set of words over some alphabet (the set of $\mathcal{L}$-sentences over $\sigma$ ) and a decidable relation $=_{\sigma}^{\mathcal{L}}$ between $\sigma$-models and $\mathcal{L}$-sentences over $\sigma$ (the truth relation, normally denoted by just $\models$ if no confusion arises). If $\varphi$ is an $\mathcal{L}$-sentence over $\sigma$, then $\operatorname{MOD}(\varphi)$ denotes the set of $\sigma$-models $\mathbf{M}$ such that $\mathbf{M} \models \varphi$. We say that $\operatorname{MOD}(\varphi)$ is defined by $\varphi$.

The notion of a logic closed e.g. under negation is defined in the natural way. We assume familiarity with commonly used logics such as first order logic $(F O)$, second order logic $(S O)$ and the prenex classes of second order logic $\left(\Sigma_{m}^{1}\right)$. In second order formulae, we sometimes use numerical superscripts to indicate the arity of relational variables - thus, $R^{r}$ is an $r$-ary variable. We also often use the same symbols to denote relations and relational variables.

If $\mathcal{L}$ and $\mathcal{L}^{\prime}$ are logics, then $\mathcal{L} \leq \mathcal{L}^{\prime}$ ( " $\mathcal{L}^{\prime}$ is at least as expressive as $\left.\mathcal{L}^{\prime \prime}\right)$ means that for any $\mathcal{L}$-sentence $\varphi$ there is an $\mathcal{L}^{\prime}$-sentence $\varphi^{\prime}$ such that $\operatorname{MOD}(\varphi)=\operatorname{MOD}\left(\varphi^{\prime}\right) . \mathcal{L} \equiv \mathcal{L}^{\prime}$ holds if $\mathcal{L} \leq \mathcal{L}^{\prime}$ and $\mathcal{L}^{\prime} \leq \mathcal{L} . \mathcal{L}<\mathcal{L}^{\prime}$ holds if $\mathcal{L} \leq \mathcal{L}^{\prime}$ but not $\mathcal{L} \equiv \mathcal{L}^{\prime}$. For any vocabulary $\sigma, \mathcal{L} \leq_{\sigma} \mathcal{L}^{\prime}$ means that for any $\mathcal{L}$-sentence $\varphi$ of vocabulary $\sigma$ there is an $\mathcal{L}^{\prime}$-sentence $\varphi^{\prime}$ such that $\operatorname{MOD}(\varphi)=\operatorname{MOD}\left(\varphi^{\prime}\right) . \mathcal{L} \equiv_{\sigma} \mathcal{L}^{\prime}$ and $\mathcal{L}<_{\sigma} \mathcal{L}^{\prime}$ are defined accordingly.

We assume familiarity with standard computational complexity classes such as $P, N P$ or $P H$. Recall that the $\Sigma_{m}^{p}$ level of $P H$ can be defined as $\bigcup_{k \in \omega} \Sigma_{m} T I M E\left(n^{k}\right)$, where $\Sigma_{m} T I M E(f)$ consists of problems solvable in time $f$ by a $\Sigma_{m}$ machine, i.e. an alternating machine which starts in an existential state and makes at most $(m-1)$ alternations between existential and universal states on any input. The same holds for $\Pi_{m}^{p}$ and the dual notion of $\Pi_{m} T I M E$.

The class $P^{N P\left[n^{r}\right]}$ consists of those problems which may be solved by a deterministic polynomial-time oracle machine which makes at most $n^{r}$ queries to an $N P$ oracle on an input of size $n$ (note that when considering 
problems defined as classes of $\sigma$-models for some $\sigma$, we take the size of a given $\sigma$-model $\mathbf{M}$ to be equal to $M$, and not to the length of a standard code for M, which may be polynomially larger). The nondeterministic exponential time class NEXPTIME is defined as $\bigcup_{k \in \omega} N T I M E\left(2^{n^{k}}\right)$.

If $\mathcal{L}$ is a logic and $\mathcal{C}$ is a complexity class, we say that $\mathcal{L}$ captures $\mathcal{C}$ if for any $\sigma$ and any class of $\sigma$-models $\mathcal{K}$, it holds that $\mathcal{K} \in \mathcal{C}$ if and only if $\mathcal{K}=\operatorname{MOD}(\varphi)$ for some $\mathcal{L}$-sentence $\varphi$. If every $\mathcal{L}$-definable class $\mathcal{K}$ (of $\sigma$-models) is in $\mathcal{C}$, we say that model checking for $\mathcal{L}$ (over $\sigma$ ) is in $\mathcal{C}$. If every class in $\mathcal{C}$ (of $\sigma$-models) is $\mathcal{L}$-definable, $\mathcal{L}$ is said to capture at least $\mathcal{C}$ (over $\sigma)^{1}$.

A well-known result is that $\Sigma_{m}^{1}$ captures $\Sigma_{m}^{p}$ for any $m$ ([F74],[Sto77]).

\subsection{FM-truth definitions}

We prove our main result using FM-truth definitions. The idea of FM-truth definitions was introduced in [MM01] and developed in [K04]. The present subsection discusses the basic definitions and results related to this concept.

Definition 1.1. A relation $R \subseteq \omega^{n}$ is $F M$-represented by the (first order) $\sigma_{0}$-formula $\varphi(\mathbf{x})$ if and only if: for any $\mathbf{a} \in \omega^{n}, \varphi(\mathbf{a})$ is true in almost all $\sigma_{0}$-models if $R(\mathbf{a})$ holds, and false in almost all $\sigma_{0}$-models if $R(\mathbf{a})$ does not hold. $R$ is $F M$-representable if there is a formula which FM-represents it.

Theorem 1.2 ([MM01]; FM-representability theorem). $R \subseteq \omega^{n}$ is FMrepresentable if and only if it is of degree $\leq \mathbf{0}^{\prime}$ (recursive with an $R E$ oracle).

The notion of FM-representability was intended as a finite model counterpart of the classical notions of definability (in a model, esp. in the standard model of arithmetic) or representability (in a theory) of arithmetical relations. One consequence of the FM-representability theorem is that, just as in the standard model of arithmetic or in arithmetical theories, also in finite models we may freely talk about operations and relations connected to the syntax of logics (such as "formula $x$ is the result of preceding formula $y$ with

\footnotetext{
${ }^{1}$ Note that it is more usual to define " $\mathcal{L}$ captures $\mathcal{C}$ " and the other related notions over models with a built-in linear ordering, and not built-in arithmetic. In our framework, some logics (such as first order logic) may capture larger complexity classes than in the usual one. However, if a logic semantically contains deterministic transitive closure logic $(D T C)$, it is able to define the arithmetical relations from the ordering, so its expressive power is the same in both frameworks.
} 
an existential quantifier) - all these relations are decidable, so we simply use the formulae which FM-represent them. Care must be taken, however, since FM-representation works "asymptotically": a formula $\varphi$ which FMrepresents some relation will only tell us whether a given tuple $\mathbf{a}$ is in the relation if we look at the truth value of $\varphi(\mathbf{a})$ in a sufficiently large model.

Henceforth, $\ulcorner w\urcorner$ stands for the Gödel number of the string $w$ (we assume that some appropriate gödelization has been carried out).

The concept of truth FM-truth definition is a finite model analogue of Tarski's notion of truth definiton:

Definition 1.3. Let $\mathcal{L}$ be a logic and $\sigma$ be a vocabulary. We say that the $\sigma$-formula $\operatorname{Tr}_{\mathcal{L}, \sigma}(x)$ is an FM-truth definition for $\mathcal{L}$ over $\sigma$ if and only if for every $\mathcal{L}$-sentence $\psi$ of vocabulary $\sigma$,

$$
\mathbf{M} \models \psi \equiv \operatorname{Tr}_{\mathcal{L}, \sigma}(\ulcorner\psi\urcorner)
$$

holds for almost all $\sigma$-models $\mathbf{M}$.

Remark. Under our definition of logic, there are logics for which the notion of a formula with a free (first order) variable does not, strictly speaking, make sense. The formal way to deal with this problem is to think of such "formulae" as sentences of vocabulary $(\sigma+c)$, where $c$ is a new individual constant. The details of a suitable redefinition of "FM-truth definition" are left to the reader.

For any logics $\mathcal{L}, \mathcal{L}^{\prime}$, if there is an $\mathcal{L}^{\prime}$-formula which is an FM-truth definition for $\mathcal{L}$ over vocabulary $\sigma$, we say that $\mathcal{L}^{\prime}$ defines FM-truth for $\mathcal{L}$ over $\sigma$ and write $\mathcal{L} \ll_{\sigma} \mathcal{L}^{\prime}$.

Using the FM-representability theorem, one may prove a finite model version of the Gödel diagonal lemma, and derive from it a version of Tarski's famous theorem on the undefinability of truth: no (reasonable) logic closed under negation defines FM-truth for itself.

Theorem 1.4 ([MM01]; Tarski's theorem, finite version). If $\mathcal{L}$ is a logic closed under first order quantification, forming conjunctions with first order formulae, and negation, then for any $\sigma$ it is not true that $\mathcal{L} \ll_{\sigma} \mathcal{L}$.

When considering one of the usual logics met in finite model theory, one can often actually give an exact characterization of the logics for which it defines FM-truth. For our current purposes, we will only need such characterizations for the $\Sigma_{m}^{1}$ and $\Pi_{m}^{1}$ classes: 
Theorem 1.5 ([K04]). For any $m, \mathcal{L}$ and $\sigma: \mathcal{L} \ll_{\sigma} \Sigma_{m}^{1}$ if and only if there exists a number $k$ such that model checking for $\mathcal{L}$ over $\sigma$ is in $\Sigma_{m} \operatorname{TIME}\left(n^{k}\right)$. Analogously for $\Pi_{m}^{1}$ and $\Pi_{m}$ TIME.

The "only if" part of theorem 1.5 is essentially trivial. The proof of the "if" part, while also simple, requires a result stating that the difficulty of defining FM-truth for a logic depends only on its expressive power, not on the peculiarities of its syntax (theorem 3.2 in [K04]).

\section{$2 P^{N P\left[n^{r}\right]}$ versus $\Sigma_{m}^{p}$}

We turn now to a proof of (A). The key observation is that for any given $r$, all $P^{N P\left[n^{r}\right]}$ properties of finite models can be defined by second order sentences in a particular normal form:

Lemma 2.1. Let $\sigma$ be a vocabulary and let $r \geq 1$. Then any $P^{N P\left[n^{r}\right]}$ class of $\sigma$-models can be defined by a sentence of the form

$$
\exists R^{r}(\varphi(R) \& \neg \psi(R)),
$$

where $\varphi, \psi$ are $\Sigma_{1}^{1}$ formulae of vocabulary $\sigma$ with $R$ as the unique free variable.

This normal form result is very similar to results proved for logics capturing relativized logarithmic space classes by I. Stewart (theorem 3.3.1 and corollary 3.3.1 of [Ste93]) and G. Gottlob (theorem 4.9 and corollary 5.3 of [G97]). The proof is also almost identical to the one given by Gottlob. We present it here in some detail so that the reader may verify that it is constructive; we will need this fact in the next section (see the proof of lemma $3.3)$.

The class of $S O$ sentences in the form given by the lemma will be denoted by $S N F_{r}$ ("SNF" stands for Stewart Normal Form, a term coined in [G97] to refer to the normal form for logics capturing relativized LOGSPACE).

Proof. Let $\mathcal{K}$ be a $P^{N P\left[n^{r}\right]}$ class of $\sigma$-models. Thus, there is a polynomialtime deterministic oracle machine $T$ and an $N P$ language $L \in\{0,1\}^{*}$ such that $\mathcal{K}=\left\{\mathbf{M}: \mathbf{M}\right.$ is a $\sigma$-model and $T_{1}$ accepts $\mathbf{M}$ using $L$ as its oracle $\}$. Furthermore, $T$ makes at most $M^{r}$ oracle queries on input $\mathbf{M}$. Thus, the string oans $(\mathbf{M})$ of all oracle answers in the computation of $T$ on input $\mathbf{M}$ 
(ordered chronologically) has length at most $M^{r}$. Recall that there is a canonical correspondence between binary strings of length $M^{r}$ and $r$-ary relations over $M$.

We introduce two classes of $\left(\sigma+R^{r}\right)$-models (where $R$ is a relational symbol not contained in $\sigma$ ). The classes $\mathcal{K}_{1}$ and $\mathcal{K}_{2}$ are defined as follows:

(1) Given $(\mathbf{M}, R)$, consider the machine $T_{R}^{+}$which behaves as $T$ except that before making its $i$-th oracle query (for $i=1, \ldots, M^{r}$ ), it looks at the $i$-th bit of the string corresponding to $R$ - and then makes a reqular query to $L$ if the bit is 1 , simulates a negative answer without querying if the bit is 0 . $(\mathbf{M}, R)$ is in $\mathcal{K}_{1}$ iff: (a) all queries regularly made by $T_{R}^{+}$on input $\mathbf{M}$ are answered positively, and (b) $T_{R}^{+}$accepts $\mathbf{M}$.

(2) Given $(\mathbf{M}, R)$, consider the machine $T_{R}^{-}$which behaves as $T$ except that before making its $i$-th oracle query (for $i=1, \ldots, M^{r}$ ), it looks at the $i$-th bit of the string corresponding to $R$ - and then makes a reqular query to $L$ if the bit is 0 , simulates a positive answer without querying if the bit is 1 . $(\mathbf{M}, R)$ is in $\mathcal{K}_{2}$ iff all queries regularly made by $T_{R}^{+}$on input $\mathbf{M}$ are answered negatively.

Consider now the second order sentence $\gamma:=\exists R^{r}(\varphi(R) \& \neg \psi(R)$ ) (where $\varphi(R)$ is $\varphi$, now treated as a formula of vocabulary $\sigma$ with $R$ as a free variable; similarly for $\psi(R))$. Certainly, $\gamma$ has the required form, so it remains to check that it defines $\mathcal{K}$.

It is fairly easy to see that $\mathcal{K}_{1}$ is in $N P$ and $\mathcal{K}_{2}$ is in co- $N P$. Let $\varphi, \psi$ be $\Sigma_{1}^{1}$ sentences of vocabulary $(\sigma+R)$ such that $\mathcal{K}_{1}=\operatorname{MOD}(\varphi), \mathcal{K}_{2}=\operatorname{MOD}(\neg \psi)$.

Consider now the second order sentence $\gamma:=\exists R^{r}(\varphi(R) \& \neg \psi(R)$ ) (where $\varphi(R)$ is $\varphi$, now treated as a formula of vocabulary $\sigma$ with $R$ as a free variable; similarly for $\psi(R))$. Certainly, $\gamma$ has the required form, so it remains to check that it defines $\mathcal{K}$.

Let $\mathbf{M} \in \mathcal{K}$ and let $R_{0} \subseteq M^{r}$ be the relation corresponding to oans $(\mathbf{M})$ (if oans $(\mathbf{M})$ is shorter than $M^{r}$, let $R_{0}$ be any relation corresponding to a string which has oans $(\mathbf{M})$ as an initial substring). Then $\left(\mathbf{M}, R_{0}\right)$ is in $\mathcal{K}_{1}$ and $\mathcal{K}_{2}$, so $\mathbf{M} \models \gamma$.

Let $\mathbf{M} \models \gamma$ and let $R \subseteq M^{r}$ be such that $(\mathbf{M}, R) \models \varphi,(\mathbf{M}, R) \models \neg \psi$. Then $(\mathbf{M}, R)$ is in both $\mathcal{K}_{1}$ and $\mathcal{K}_{2}$, so, by an inductive argument, the string corresponding to $R$ either simply is oans $(\mathbf{M})$ or contains oans $(\mathbf{M})$ as an initial substring. In any case, $T_{R}^{+}$works on input $\mathbf{M}$ exactly as $T$ does, and since $(\mathbf{M}, R) \in \mathcal{K}_{1}$, we know that $T_{R}^{+}$accepts $\mathbf{M}$. Then so does $T$, and therefore $\mathbf{M}$ is in $\mathcal{K}$

Thus, the proof that $\gamma$ defines $\mathcal{K}$ is completed. 
Once we have the above lemma, the proof of (A) presents no further difficulty. Fix $m$ and assume that the hypothesis of (A) holds for $m$. It follows from this assumption and from theorem 1.5 that for any vocabulary $\sigma, \Sigma_{1}^{1} \ll_{\sigma} \Sigma_{m}^{1}, \Sigma_{1}^{1} \ll_{\sigma} \Pi_{m}^{1}$.

Fix $r$ and a vocabulary $\sigma$. Let $\operatorname{Sat}_{\Sigma}(x)\left(\right.$ resp. $\left.\operatorname{Sat}_{\Pi}(x)\right)$ be a $\Sigma_{m}^{1}($ resp. $\left.\Pi_{m}^{1}\right)$ FM-truth definition for $\Sigma_{1}^{1}$ over the vocabulary $\left(\sigma+R^{r}\right)$. Consider the following formula $\operatorname{Tr}(x)$ :

$$
\exists\ulcorner\varphi\urcorner \exists\ulcorner\psi\urcorner\left(x=\left\ulcorner\exists R^{r}(\varphi(R) \& \neg \psi(R))\right\urcorner \& \exists R^{r}\left(\operatorname{Sat}_{\sigma}(\ulcorner\varphi\urcorner) \& \neg \operatorname{Sat}_{\Pi}(\ulcorner\psi\urcorner)\right)\right) .
$$

$\operatorname{Tr}(x)$ is clearly equivalent to a $\Sigma_{m}^{1}$ formula, and one may easily verify that it is an FM-truth definition for $S N F_{r}$ over $\sigma$.

Thus, again by theorem 1.5, for every $r$ and $\sigma$ there exists a number $k_{r}$ such that $P^{N P\left[n^{r}\right]} \subseteq \Sigma_{m}$ TIME $\left(n^{k_{r}}\right)$ over $\sigma$. In particular, such $k_{r}$ exists for the vocabulary $\left(\sigma_{0}+P^{1}\right)$ where $P^{1}$ is some chosen unary predicate. Note that models of this vocabulary can be identified with binary words. Thus, $P^{N P\left[n^{r}\right]} \subseteq \Sigma_{m} T I M E\left(n^{k_{r}}\right)$ as classes of languages over $\{0,1\}$.

The inclusion $P^{N P\left[n^{r}\right]} \subseteq \Pi_{m} T I M E\left(n^{k_{r}}\right)$ follows from the closure of $P^{N P\left[n^{r}\right]}$ under complementation.

\section{$3 \quad P^{N P\left[n^{r}\right]}$ versus $\Sigma_{m}^{p} \cap \prod_{m}^{p}$}

The proof of $(\mathrm{B})$ is quite similar to the proof of $(\mathrm{A})$, although it requires some more care. We introduce a logic $\mathfrak{L}_{r}$ which captures $P^{N P\left[n^{r}\right]}$, and show that if $\Sigma_{m}^{p} \cap \Pi_{m}^{p}$ contains NTIME(f) for a superpolynomial time-constructible $f$, then for every vocabulary $\sigma, \mathfrak{L}_{r} \ll_{\sigma} \Delta_{m}^{1}$ (i.e. there is a $\Sigma_{m}^{1}$ FM-truth definition for $\mathfrak{L}_{r}$ which is equivalent to a $\Pi_{m}^{1}$ formula). (B) will then follow by Tarski's theorem.

Remark. $\Delta_{m}^{1}$ is not necessarily a logic, even under the liberal notion of section 1 , so it is a slight abuse of notation to write $\mathfrak{L}_{r} \ll_{\sigma} \Delta_{m}^{1}$. Nevertheless, it remains true that if there is a $\Delta_{m}^{1}$ FM-truth definition for some $\mathcal{L}$ over some vocabulary $\sigma$, then there is a $\Sigma_{m}^{p} \cap \Pi_{m}^{p}$ property which is not $\mathcal{L}$-definable.

The logic $\mathfrak{L}_{r}$ we will use is defined as follows. For any vocabulary $\sigma$, the $\mathfrak{L}_{r}$-sentences over $\sigma$ are ordered pairs $\left\langle T_{1}, T_{2}\right\rangle$, where: 
- $T_{1}$ is a (code of a) deterministic oracle machine, equipped with a polynomial time clock and a query counter which prohibits $T_{1}$ from asking more than $n^{r}$ oracle queries on inputs of size $n$;

- $T_{2}$ is a (code of a) nondeterministic Turing machine equipped with a polynomial time clock.

The semantics is straightforward: $\mathbf{M} \models\left\langle T_{1}, T_{2}\right\rangle$ iff $T_{1}$ accepts $\mathbf{M}$ when using the language recognized by $T_{2}$ as its oracle.

Obviously, $\mathfrak{L}_{r}$ captures exactly $P^{N P\left[n^{r}\right]}$. Note that it follows from theorem 1.5 that already if the hypothesis of (A) holds for a given $m$, we have $\mathfrak{L}_{r} \ll_{\sigma}$ $\Sigma_{m}^{1}, \mathfrak{L}_{r} \ll_{\sigma} \Pi_{m}^{1}$ for every $\sigma$. To obtain $\mathfrak{L}_{r} \ll_{\sigma} \Delta_{m}^{1}$, however, we will need the (apparently) stronger assumption that $\Sigma_{m}^{p} \cap \Pi_{m}^{p}$ contains a superpolynomial nondeterministic time class.

We will also need a simple but important observation on FM-representing computable relations (or, more generally, recursively enumerable relations). Given a relation $R \subseteq \omega^{n}$ and a formula $\varphi_{R}(\mathbf{x})$ which FM-represents it, call $\varphi_{R}$ decent if it has the property that: (a) for any $\mathbf{a} \in \omega^{n}$, if there exists a model $\mathbf{M}$ such that $\mathbf{M} \models \varphi_{R}(\mathbf{a})$, then $\mathbf{a} \in R$, and (b) if $\mathbf{M} \models \varphi_{R}(\mathbf{a})$, then also $\mathbf{M}^{\prime} \models \varphi_{R}(\mathbf{a})$ whenever $M^{\prime}>M$. Thus, a decent formula is one which "never falsely claims" that some tuple is in the relation it FM-represents, and additionally "never withdraws such a claim" when passing to larger models. The observation is:

Proposition 3.1. For any recursively enumerable relation $R \subseteq \omega^{n}$, there is a decent formula which FM-represents it.

Proof. This fact is implicitly contained in the proof of the FM-representability theorem in [MM01]; we give an explicit argument nonetheless. If $R \subseteq \omega^{n}$ is $\mathrm{RE}$, then it is defined in $\mathbb{N}$ by a $\Sigma_{1}^{0}$ formula, i.e. an FO-formula of the form $\exists \mathbf{y} \psi(\mathbf{x}, \mathbf{y})$ where $\psi$ consists of a string of bounded quantifiers followed by a quantifier-free matrix. It is well-known that we may assume w.l.o.g. that the quantifiers in $\psi$ are bounded by variables and not by complex terms (see e.g. [HP93]).

The matrix of $\psi$ is a boolean combination of equalities between some polynomials in $\mathbf{x}, \mathbf{y}$, and $\mathbf{z}$, where $\mathbf{z}$ is the tuple of variables which are quantified in $\psi$. Therefore, since the $z$ 's are bounded from above by the $x$ 's and/or $y$ 's, there is a polynomial $p(\mathbf{x}, \mathbf{y})$ such that for any choice of tuples $\mathbf{a}, \mathbf{b}$ to interpret $\mathbf{x}, \mathbf{y}$ respectively, the truth of $\psi(\mathbf{a}, \mathbf{b})$ does not depend on any number greater than $p(\mathbf{a}, \mathbf{b})$. 
Let $\varphi_{R}(\mathbf{x})$ be $\exists \mathbf{y} \xi(\mathbf{x}, \mathbf{y})$, where $\xi$ is $\psi$ modified in the following way: the original quantifier prefix of $\psi$ is left unchanged, and the matrix is replaced by the conjunction of an FO-formula expressing "there exists $p(\mathbf{x}, \mathbf{y})$ " and a formula which arises from the original matrix by eliminating all the complex terms (i.e. substituting $\exists w\left(+\left(x_{1}, x_{2}, w\right) \& w=x_{3}\right)$ for $x_{1}+x_{2}=x_{3}$ etc.). It is not difficult to see that $\varphi_{R}$ is a decent formula which FM-represents $R$.

Actually, one can show that being FM-represented by some decent formula is exactly equivalent to being RE. We leave out the (easy) proof of this fact as we do not need it.

We now prove a lemma which states that if the hypothesis of (B) holds for $m$, then there exist $\Sigma_{m}^{1}$ and $\Pi_{m}^{1}$ FM-truth definitions for $\Sigma_{1}^{1}$ which are, in a sense, well-behaved.

Lemma 3.2. If $\Sigma_{m}^{p} \cap \Pi_{m}^{p}$ contains NTIME(f) for some superpolynomial time-constructible $f$, then for any vocabulary $\sigma$ there exists a $\Sigma_{m}^{1}$ formula $\operatorname{Sat}_{\Sigma}^{+}(x)$ and a $\Pi_{m}^{1}$ formula $\operatorname{Sat}_{\Pi}^{+}(x)$ such that:

- $\operatorname{Sat}_{\Sigma}^{+}(x)$ and $\operatorname{Sat}_{\Pi}^{+}(x)$ are FM-truth definitions for $\Sigma_{1}^{1}$ over $\sigma$;

- there is a computable function which assigns to a $\Sigma_{1}^{1}$ sentence $\psi$ over $\sigma$ a number bound $(\psi)$ such that both Sat $_{\Sigma}^{+}$and Sat $t_{\Pi}^{+}$recognize the truth value of $\psi$ correctly in all $\sigma$-models of cardinality greater than bound $(\psi)$.

Proof. Fix $\sigma$. From now on, any model $\mathbf{M}$ appearing in the proof is a $\sigma-$ model.

We may assume w.l.o.g. that $f(n)$ is of the form $n^{g(n)}$, where $g$ is some computable function satisfying $\lim _{n \rightarrow \infty} g(n)=\infty$; if not, then we may find such $g$ for which $n^{g(n)} \leq f(n)$ - here it is not required for $n^{g(n)}$ to be timeconstructible, computability will suffice.

Clearly, there exists a computable function $h$ such that $g(h(n)) \geq n$ for all $n$.

It is well-known that there exists a Turing machine $T$ which solves the problem:

"given input $(\mathbf{M},\ulcorner\psi\urcorner)$, where $\psi$ is a $\Sigma_{1}^{1}$ sentence, is it true that $\mathbf{M} \models \psi$ ?"

in nondeterministic time $M^{l h(\psi)}$. So, the machine $\tilde{T}$ which, given input $(\mathbf{M},\ulcorner\psi\urcorner 0 i\urcorner)$, disregards the string $0^{i}$ and simulates $T$ on $(\mathbf{M},\ulcorner\psi\urcorner)$ actually 
works in $N T I M E\left(M^{g\left(l h\left(\psi^{\curvearrowright} 0^{i}\right)\right)}\right)$, and hence in NTIME $(f)$, when restricted to inputs of the form $\left.\left(\mathbf{M},\ulcorner\psi\urcorner 0^{h(l h(\psi))-l h(\psi)}\right\urcorner\right)$.

Since the function $h$ is computable, then by proposition 3.1 there is a decent formula $\varphi_{h}(x, y)$ which FM-represents its graph. Consider now a machine $T^{*}$ which on input $\left.\left(\mathbf{M},\ulcorner\psi\urcorner 0^{i}\right\urcorner\right)$ does the following:

- using the time-constructibility of $f$, keep a clock for, say, $2 f$; if a computation tries to use more than this allotted amount of time, stop and reject;

- check whether $\mathbf{M}=\left(\varphi_{h}(\operatorname{lh}(\psi), i+\operatorname{lh}(\psi))\right.$; if not, reject;

- else work as $\tilde{T}$ on the input and accept iff $\tilde{T}$ does.

Clearly, for all sufficiently large $\mathbf{M}$, the machine $T^{*}$ accepts $\left.\left(\mathbf{M},\ulcorner\psi\urcorner 0^{i}\right\urcorner\right)$ if and only if $i=h(l h(\psi))-\operatorname{lh}(\psi)$ and $\mathbf{M} \models \psi$. Moreover, $T^{*}$ works in $N T I M E(O(f)) \subseteq N T I M E(f) \subseteq \Sigma_{m}^{p} \cap \Pi_{m}^{p}$, so there is a $\Sigma_{m}^{1}$ formula $\varphi_{\Sigma}^{*}(x)$ - equivalent to a $\Pi_{m}^{1}$ formula $\varphi_{\Pi}^{*}(x)$ - which is true of $w$ in $\mathbf{M}$ iff $T^{*}$ accepts $(\mathbf{M}, w)$.

Let $\operatorname{Sat}_{\Sigma}^{+}(x)$ be

$$
\begin{gathered}
\exists w(" w=\ulcorner x\urcorner z\urcorner \text { where } z \text { is a string of zeroes" } \\
\left.\& \varphi_{h}(\operatorname{lh}(x), \operatorname{lh}(w)) \& \varphi_{\Sigma}^{*}(w)\right),
\end{gathered}
$$

and let $\left.\operatorname{Sat}_{\Pi}^{+}(x)\right)$ be defined analogously using $\varphi_{\Pi}^{*}$. It is not hard to see that $\operatorname{Sat}_{\Sigma}^{+}(x)$ and $\operatorname{Sat}_{\Pi}^{+}(x)$ are FM-truth definitions for $\Sigma_{1}^{1}$ over $\sigma$. It therefore remains to check that given $\psi$, we can compute bound $(\psi)$ such that $\operatorname{Sat}_{\Sigma}^{+}(x)$ (or, equivalently, $\operatorname{Sat}_{\Pi}^{+}(x)$ ) works properly for $\psi$ in all models larger than bound $(\psi)$.

Before we describe the algorithm, we note that checking whether $\mathbf{M} \models$ $\left(\varphi_{h}(l h(\psi), i+l h(\psi))\right.$ on input $\left.\left(\mathbf{M},\ulcorner\psi\urcorner 0^{i}\right\urcorner\right)$ requires (deterministic) time $M^{l}$ for some fixed $l$ independent of $\psi$. By assumption, $f$ is superpolynomial, so there is a $c$ such that for all $n \geq c, f(n) \geq n^{l}$.

Now compute bound $(\psi)$ on input $\psi$ as follows. Find the smallest model $\mathbf{M}^{\prime}$ in which there is a $w$ such that $\mathbf{M}^{\prime} \models \chi(w)$, where $\chi(w)$ is

$$
\text { " } w=\ulcorner\psi\urcorner z\urcorner \text { where } z \text { is a string of zeroes" \& } \varphi_{h}(\operatorname{lh}(\psi), \operatorname{lh}(w)) .
$$

Since we may assume that both the conjuncts in $\chi$ are decent, this $w$ will be equal to $\left.\ulcorner\psi\urcorner 0^{h(l h(\psi))-l h(\psi)}\right\urcorner$ and will also satisfy $\chi$ in all models larger than $\mathbf{M}^{\prime}$. Let $\operatorname{bound}(\psi):=\max \left(M^{\prime}, c\right)$. 
Given any $(\mathbf{M}, w)$ with $M \geq \operatorname{bound}(\psi)$, the machine $T^{*}$ will recognize that $w$ is of the proper length in time $M^{l} \leq f(M)$, and check whether $\tilde{T}$ accepts $(\mathbf{M}, w)$ in time $f(M)$. Thus, $T^{*}$ will not exceed its time limit $2 f(M)$, so it will correctly answer whether $\mathbf{M} \models \psi$, which proves that bound $(\psi)$ is indeed large enough.

We need one more observation: two important constructions associated with the logic $\mathfrak{L}_{r}$ are effective and can therefore be FM-represented by decent formulae.

Lemma 3.3. There exist computable functions:

(a) neg, which assigns to (the Gödel number of) an $\mathfrak{L}_{r}$-sentence $\xi$ (the Gödel number of) the "negation" of $\psi$, i.e. an $\mathfrak{L}_{r}$-sentence neg( $\left.\xi\right)$ which is true in exactly those models in which $\xi$ is false;

(b) snf, which assigns to (the Gödel number of) an $\mathfrak{L}_{r}$-sentence $\xi$ (the Gödel number of ) a $\Sigma_{2}^{1}$ sentence sn $f(\xi)$ in $S N F_{r}$ such that $\xi$ and $\operatorname{sn} f(\xi)$ are equivalent.

Proof. For (a), let $\xi=\left\langle T_{1}, T_{2}\right\rangle$. Then $n e g(\ulcorner\xi\urcorner)$ is $\left\ulcorner\left\langle\overline{T_{1}}, T_{2}\right\rangle\right\urcorner$, where $\overline{T_{1}}$ is $T_{1}$ with accepting and rejecting states interchanged.

For (b), we just need to make sure that the construction in the proof of lemma 2.1 can be carried out in an effective way. We sketch the argument. Given $\ulcorner\xi\urcorner=\left\ulcorner\left\langle T_{1}, T_{2}\right\rangle\right\urcorner$, we may effectively construct $N P$-machines $T_{\mathcal{K}_{1}}, T_{\mathcal{K}_{2}}$ which recognize $\mathcal{K}_{1}$ and the complement of $\mathcal{K}_{2}$, respectively. Moreover, we can compute a time bound for both of these machines: if $T_{1}$ has an $n^{k_{1}}$ clock and $T_{2}$ has an $n^{k_{2}}$ clock, then $T_{\mathcal{K}_{1}}$ essentially runs as $T_{1}$ except that it will have to simulate $T_{2}$ at most $n^{r}$ times (the exact number depends on the additional input relation $R$ ) on strings of length $\leq n^{k_{1}}$ (since $T_{1}$ will not have the time to write any longer strings on its oracle tape). So a rough estimate of the time needed by $T_{\mathcal{K}_{1}}$ is $n^{k_{1}}+n^{r+k_{1} \cdot k_{2}}$. Some additional time is needed for looking at the bits of $R$, so we may take $n^{r+k_{1} \cdot k_{2}+1}$ as a suitable upper bound. The same bound will do for $T_{\mathcal{K}_{2}}$.

Once $T_{\mathcal{K}_{1}}, T_{\mathcal{K}_{2}}$, and their time bounds are known, we can use them to compute the $\Sigma_{1}^{1}$ sentences $\varphi, \psi$ which describe the action of $T_{\mathcal{K}_{1}}$ and $T_{\mathcal{K}_{2}}$, respectively. We then set $\gamma:=\exists R^{r}(\varphi(R) \& \neg \psi(R))$ and $\operatorname{sn} f(\ulcorner\xi\urcorner):=\ulcorner\gamma\urcorner$.

By proposition 3.1, there exist decent formulae which represent the graphs of neg and snf. Choose some such formulae and call them neg(x,y) and $\operatorname{snf}(x, y)$, respectively. 
We are now ready to give a proof of (B). Fix $m$ and assume that the hypothesis of (B) holds for $m$. Fix $r$ and $\sigma$. Let $\operatorname{Sat}_{\Sigma}^{+}(x)$ and $\operatorname{Sat}_{\Pi}^{+}(x)$ be the equivalent $\Sigma_{m}^{1}$ and $\Pi_{m}^{1} \mathrm{FM}$-truth definitions for $\Sigma_{1}^{1}$ over $\left(\sigma+R^{r}\right)$ given by lemma 3.2. Let bound be the function from that lemma appropriate for $\mathrm{Sat}_{\Sigma}^{+}$and $\mathrm{Sat}_{\Pi}^{+}$.

Recall that bound is a computable function. Thus, by a minor modification of the proof of proposition 3.1, there is a first order $\sigma_{0}$-formula large $(x)$ which satisfies the following three requirements for any given $\Sigma_{1}^{1}$ sentence $\psi$ over $\left(\sigma+R^{r}\right)$ :

- $\mathbf{M} \models \operatorname{large}(\ulcorner\psi\urcorner)$ implies $M \geq \operatorname{bound}(\psi)$;

- $\mathbf{M} \models \operatorname{large}(\ulcorner\psi\urcorner)$ for all sufficiently large $\mathbf{M}$;

- if $\mathbf{M} \models \operatorname{large}(\ulcorner\psi\urcorner)$ and $M^{\prime} \geq M$, then $\mathbf{M}^{\prime} \models \operatorname{large}(\ulcorner\psi\urcorner)$.

Consider now the formula $\operatorname{good}\left(x, x^{\prime}, y, y^{\prime},\ulcorner\varphi\urcorner,\ulcorner\psi\urcorner,\left\ulcorner\varphi^{\prime}\right\urcorner,\left\ulcorner\psi^{\prime}\right\urcorner\right)$ :

$$
\begin{gathered}
\operatorname{neg}\left(x, x^{\prime}\right) \& \operatorname{snf}(x, y) \& \operatorname{snf}\left(x^{\prime}, y^{\prime}\right) \\
\& y=\left\ulcorner\exists R^{r}(\varphi(R) \& \neg \psi(R))\right\urcorner \& y^{\prime}=\left\ulcorner\exists R^{r}\left(\varphi^{\prime}(R) \& \neg \psi^{\prime}(R)\right)\right\urcorner \\
\text { \& large }(\ulcorner\varphi\urcorner) \& \operatorname{large}(\ulcorner\psi\urcorner) \& \operatorname{large}\left(\left\ulcorner\varphi^{\prime}\right\urcorner\right) \& \operatorname{large}\left(\left\ulcorner\psi^{\prime}\right\urcorner\right) .
\end{gathered}
$$

Let $\operatorname{Tr}_{\Sigma}(x)$ be:

$$
\exists x^{\prime} \ldots \exists\left\ulcorner\psi^{\prime}\right\urcorner\left(\operatorname{good}\left(x, x^{\prime}, \ldots,\left\ulcorner\psi^{\prime}\right\urcorner\right) \& \exists R^{r}\left(\operatorname{Sat}_{\Sigma}^{+}(\ulcorner\varphi\urcorner) \& \neg S a t_{\Pi}^{+}(\ulcorner\psi\urcorner)\right)\right),
$$

and let $\operatorname{Tr}_{\Pi}(x)$ be:

$$
\exists x \ldots \exists\left\ulcorner\psi^{\prime}\right\urcorner\left(\operatorname{good}\left(x, x^{\prime}, \ldots,\left\ulcorner\psi^{\prime}\right\urcorner\right) \& \neg \exists R^{r}\left(\operatorname{Sat}_{\Sigma}^{+}\left(\left\ulcorner\varphi^{\prime}\right\urcorner\right) \& \neg S a t_{\Pi}^{+}\left(\left\ulcorner\psi^{\prime}\right\urcorner\right)\right)\right) .
$$

It is not hard to see that $\operatorname{Tr}_{\Sigma}$ is equivalent to a $\Sigma_{m}^{1}$ formula, that $\operatorname{Tr}_{\Pi}$ is equivalent to a $\Pi_{1}^{1}$ formula, and that both $\operatorname{Tr}_{\Sigma}$ and $\operatorname{Tr}_{\Pi}$ are FM-truth definitions for $\mathfrak{L}_{r}$ over $\sigma$. Let us then check that $\operatorname{Tr}_{\Sigma}$ and $\operatorname{Tr}_{\Pi}$ are equivalent.

It may be assumed that neither $\operatorname{Tr}_{\Sigma}(w)$ nor $\operatorname{Tr}_{\Pi}(w)$ ever holds if $w$ is not the Gödel number of an $\mathfrak{L}_{r}$-sentence. So let $\mathbf{M}$ be a $\sigma$-model and let $\ulcorner\xi\urcorner \in M$ for some $\mathfrak{L}_{r}$-sentence $\xi$. If there is no tuple $\left\langle x^{\prime}, \ldots,\left\ulcorner\psi^{\prime}\right\urcorner\right\rangle$ such that $\mathbf{M} \models \operatorname{good}\left(\ulcorner\xi\urcorner, x^{\prime}, \ldots,\left\ulcorner\psi^{\prime}\right\urcorner\right)$, then we have $\mathbf{M} \not \models \operatorname{Tr}_{\Sigma}(\ulcorner\xi\urcorner)$ and $\mathbf{M} \not \models$ $\operatorname{Tr}_{\Pi}(\ulcorner\xi\urcorner)$.

Otherwise, by the decency of the formulae neg and $s n f$, it must be the case that $x^{\prime}=\operatorname{neg}(\ulcorner\xi\urcorner)$, that $y=\operatorname{snf}(\ulcorner\xi\urcorner)=\left\ulcorner\exists R^{r}(\varphi(R) \& \neg \psi(R))\right\urcorner$, and 
that $y^{\prime}=\operatorname{sn} f(n e g(\ulcorner\xi\urcorner))=\left\ulcorner\exists R^{r}\left(\varphi^{\prime}(R) \& \neg \psi^{\prime}(R)\right)\right\urcorner$. Moreover, by the choice of the formula large, $M$ is large enough for $S a t_{\Sigma}^{+}$and $S a t_{\Pi}^{+}$to correctly recognize, given any $R \subseteq M^{r}$, the truth value of $\varphi, \psi, \varphi^{\prime}$, and $\psi^{\prime}$ in $(\mathbf{M}, R)$. Thus, $\mathbf{M} \models \operatorname{Tr}_{\Sigma}(\ulcorner\xi\urcorner)$ iff $\mathbf{M} \models \exists R^{r}(\varphi(R) \& \neg \psi(R))$, that is, iff $\mathbf{M} \models \xi$. Similarly, $\mathbf{M} \models \operatorname{Tr}_{\Pi}(\ulcorner\xi\urcorner)$ iff $\mathbf{M} \forall \forall \exists R^{r}\left(\varphi^{\prime}(R) \& \neg \psi^{\prime}(R)\right)$, that is, again, iff $\mathbf{M} \models \xi$. So, also in this case $\mathbf{M} \models \operatorname{Tr}_{\Sigma}(\ulcorner\xi\urcorner)$ iff $\mathbf{M} \models \operatorname{Tr}_{\Pi}(\ulcorner\xi\urcorner)$, which proves that $\operatorname{Tr}_{\Sigma}$ and $T r_{\Pi}$ are indeed equivalent.

Hence, for any choice of $r$ and $\sigma$, we have found a $\Delta_{m}^{1}$ FM-truth definition for $\mathfrak{L}_{r}$ over $\sigma$. By the finite version of Tarski's theorem, for any $r$ and over any $\sigma$ which contains an individual constant, it holds that $\mathfrak{L}_{r}$ is strictly less expressive that $\Delta_{m}^{1}$, so $P^{N P\left[n^{r}\right]}$ is properly contained in $\Sigma_{m}^{p} \cap \Pi_{m}^{p}$. It follows that $P^{N P\left[n^{r}\right]} \subsetneq \Sigma_{m}^{p} \cap \Pi_{m}^{p}$ as classes of languages over $\{0,1\}$.

\section{Concluding remarks}

I. There are no obstacles to extending the methods of sections 2 and 3 to $P^{\Sigma_{i}^{p}\left[n^{r}\right]}$ instead of just $P^{N P\left[n^{r}\right]}$. Thus, for any $i$, we obtain the following result:

Theorem 4.1. Let $m \geq i+1$. Then:

(A) If there exists $k$ such that $\Sigma_{i}^{p} \subseteq \Sigma_{m} T I M E\left(n^{k}\right) \cap \Pi_{m} T I M E\left(n^{k}\right)$, then for every $r$ there exists $k_{r}$ such that $P^{\Sigma_{i}^{p}\left[n^{r}\right]} \subseteq \Sigma_{m} T I M E\left(n^{k_{r}}\right) \cap \Pi_{m} T I M E\left(n^{k_{r}}\right)$;

(B) If there exists a superpolynomial time-constructible function $f$ such that $\Sigma_{i} T I M E(f) \subseteq \Sigma_{m}^{p} \cap \Pi_{m}^{p}$, then additionally $P^{\Sigma_{i}^{p}\left[n^{r}\right]} \subsetneq \Sigma_{m}^{p} \cap \Pi_{m}^{p}$.

II. Recall that $L O G S P A C E^{N P}$ is $P^{N P[O(\log n)]}$ ([W90],[BH91]). Thus, for example, if there exists $k$ such that $N P \subseteq \Sigma_{m} T I M E\left(n^{k}\right) \cap \Pi_{m} T I M E\left(n^{k}\right)$, then there also is some $k^{\prime}$ such that LOGSPACE $E^{N P} \subseteq \Sigma_{m} T \operatorname{IME}\left(n^{k^{\prime}}\right) \cap$ $\Pi_{m} T I M E\left(n^{k^{\prime}}\right)$. Obviously, appropriate analogues of part (B) of theorem 0.1 , and of theorem 4.1, also hold.

III. Define $\left(\Sigma_{1}^{1}\right)^{\leq r}$ as the subclass of $\Sigma_{1}^{1}$ consisting of those formulae in which the existential second order quanitifiers have arity at most $r$. The hierarchy $\left\langle\left(\Sigma_{1}^{1}\right)^{\leq r}\right\rangle_{r \in \omega}$ is sometimes referred to as the $\Sigma_{1}^{1}$ arity hierarchy. This hierarchy is known to be strict if we take into account vocabularies of arbitrary arity ([A83]), but its strictness over a uniform vocabulary remains an open problem.

Observe that if model checking for $F O$ over any fixed vocabulary is contained in NTIME $\left(n^{k}\right)$ - the number $k$ may depend on $\sigma$ - then there is 
a $\Sigma_{1}^{1}$ FM-truth definition for $F O$ over any vocabulary $\sigma$. It is a routine task to transform these definitions into $\Sigma_{1}^{1} F M$-truth definitions for $\left(\Sigma_{1}^{1}\right)^{\leq r}$, for any $r$ and over any $\sigma$. We therefore have:

Proposition 4.2. If for any $\sigma$ there is $k$ such that model checking for FO over $\sigma$ is in NTIME $\left(n^{k}\right)$, then for any $\sigma$ and $r$ there is $k_{r}$ such that model checking for $\left(\Sigma_{1}^{1}\right)^{\leq r}$ over $\sigma$ is in NTIME $\left(n^{k_{r}}\right)$ (and thus the $\Sigma_{1}^{1}$ arity hierarchy is infinite - hence, by [F75], strict - over any $\sigma)$.

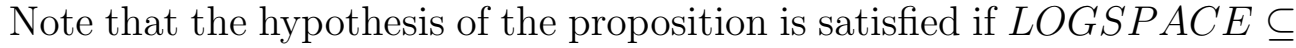
$N T I M E\left(n^{k}\right)$ for some $k$, in particular if $N P$ contains $D S P A C E(f)$ for any space-constructible function $f$ which dominates $l o g$.

Again, this can be generalized to arity hierarchies of the higher prenex classes of $S O$. Let $\left(\Sigma_{m}^{1}\right)^{\leq r}$ denote the subclass of $\Sigma_{m}^{1}$ consisting of formulae in which the second order quantifiers in the initial existential quantifier block have arity at most $r$ (the arity of the other relational quantifiers is arbitrary). We have:

Proposition 4.3. If there is $k$ such that $\Pi_{m-1}^{p} \subseteq \Sigma_{m} T I M E\left(n^{k}\right)$, then for $\sigma$ and $r$ there is $k_{r}$ such that model checking for $\left(\Sigma_{m}^{1}\right)^{\leq r}$ over $\sigma$ is in NTIME $\left(n^{k_{r}}\right)$.

Note again that the hypothesis will hold e.g. if $\Sigma_{m}^{p}$ contains $\Pi_{m-1} T I M E(f)$ for a time-constructible superpolynomial $f$.

IV. As pointed out in the introduction, our theorem 0.1 has as its consequence the (already known) result that for any $r, P^{N P\left[n^{r}\right]} \subsetneq N E X P T I M E$. It is perhaps worth noting that the largest syntactically defined fragments of $S O$ which can be separated from NEXPTIME by a similar argument are the closures of $\Sigma_{1}^{1}$ under second order quantifiers of bounded arity. For, let $S O \leq r\left(\Sigma_{1}^{1}\right)$ denote the closure of $\Sigma_{1}^{1}$ under boolean connectives, first order quantification, and second order quantification over relations of arity at most $r$. Then we easily get:

Proposition 4.4. If $N P \subseteq D S P A C E\left(n^{k}\right)$ for some $k$, then for any $\sigma$ there exists $k^{\prime}$ such that all $S O \leq r\left(\Sigma_{1}^{1}\right)$-definable classes of $\sigma$-models are in $D S P A C E\left(n^{k^{\prime}}\right)$,

from which it follows that $S O^{\leq r}\left(\Sigma_{1}^{1}\right)$ cannot define all NEXPTIME classes of models. 
V. We proved part (B) of theorem 0.1 by showing that if the hypothesis of this part holds, then there is a $\Delta_{m}^{1}$ FM-truth definition for the logic $\mathfrak{L}_{r}$ (over any vocabulary). Compared to the construction of $\Sigma_{m}^{1}$ and $\Pi_{m}^{1} \mathrm{FM}-$ truth definitions in the proof of part (A), the construction of a $\Delta_{m}^{1}$ definition required some more subtlety - and an additional assumption.

This suggests the following, possibly interesting, question: given a vocabulary $\sigma$, is there any natural characterization of the logics for which $\Delta_{m}^{1}$ can define FM-truth over $\sigma$ ? Clearly, for any such logic $\mathcal{L}$ there must exist some $k$ such that model checking for $\mathcal{L}$ over $\sigma$ is contained in $\Sigma_{m} T \operatorname{IME}\left(n^{k}\right) \cap$ $\Pi_{m} T I M E\left(n^{k}\right)$. However, there appears to be no good reason to suspect that this is sufficient, as the $\Sigma_{m}^{1}$ and $\Pi_{m}^{1}$ FM-truth definitions whose existence follows by theorem 1.5 from the existence of such a number $k$ need not be equivalent.

\section{References}

[A83] M. Ajtai, $\Sigma_{1}^{1}$ Sentences on Finite Structures, in Annals of Pure and Applied Logic 24(1983), pp. 1-48.

[BFFT01] H. Buhrman, S. Fenner, L. Fortnow and L. Torenvliet, Two Oracles that Force a Big Crunch, in Computational Complexity 10(2001), pp. 93-116.

[BH91] S. R. Buss and L. HAY, On Truth-Table Reducibility to SAT, in Information and Computation 91(1991), pp. 86-102.

[BT94] H. Buhrman and L. Torenvliet, On the Cutting Edge of Relativization: The Resource Bounded Injury Method, in Proc. International Colloquium on Automata, Languages and Programming 1994, Springer-Verlag 1994, pp. 263-273.

[F74] R. FAGin, Generalized First-Order Spectra and Polynomial-Time Recognizable Sets, in Complexity of Computation, SIAM-AMS Proceedings 7(1974), pp. 43-73.

[F75] R. FAGIN, A Spectrum Hierarchy, in Zeitschrift für Mathematische Logik und Grundlagen der Mathematik 21(1975), pp. $123-134$. 
[FLZ92] B. Fu, H. Li, and Y. Zhong, Some Properties of Exponential Time Complexity Classes, in Proc. Structure in Complexity Theory Conference 1992, IEEE Computer Society Press 1992, pp. 50-57.

[G97] G. Gotтlob, Relativized Logspace and Generalized Quantifiers over Finite Ordered Structures, Journal of Symbolic Logic 62(1997), pp. 545-574.

[HP93] P. HÁJeK and P. PUdLÁK, Metemathematics of First-Order Arithmetic, Springer-Verlag 1993.

[K04] L. A. KoŁodziejczyk, Truth Definitions in Finite Models, in Journal of Symbolic Logic 69(2004), pp. 183-200.

[M96] S. MocAs, Separating Classes in the Exponential-Time Hierarchy From Classes in PH, in Theoretical Computer Science 158(1996), pp. 221-231.

[MM01] M. Mostowski, On Representing Concepts in Finite Models, in Mathematical Logic Quarterly 47(2001), pp. 513-523.

[Ste93] I. A. Stewart, Logical Characterization of Bounded Query Classes I: Logspace Oracle Machines, in Fundamenta Informaticae 18(1993), pp. 65-92.

[Sto77] L. Stockmeyer, The Polynomial-Time Hierarchy, in Theoretical Computer Science 3(1977), pp. 1-22.

[W90] K. W. Wagner, Bounded Query Classes, in SIAM Journal on Computing 19(1990), pp. 833-846. 